UDC 533.9

DOI: $10.22363 / 2658-4670-2019-27-2-133-142$

\title{
On the radiation losses during motion of an electron in the field of intense laser radiation
}

\author{
Ekaterina V. Dobrova, Vladimir P. Milantiev \\ Institute of Physical Research and Technology \\ Peoples' Friendship University of Russia (RUDN university) \\ 6, Miklukho-Maklaya St., Moscow, 117198, Russian Federation
}

(received: April 20, 2019; accepted: November 14, 2019)

\begin{abstract}
Motion of the relativistic electron in the field of intense laser pulse of the arbitrary shape is considered. The pulse dimension is supposed to be of the order of the Gaussian laser beam dimension in the focal plane. It is supposed that the pulse is propagating along the external constant magnetic field. In the paraxial approximation the corrections of the first order to the vectors of the field of radiation as well as the force of the radiation friction are taken into account. Averaged relativistic equations of motion of electron are obtained with the help of averaging over the fast oscillations of the laser radiation. It is shown that with taking into account corrections of the first order to the field vectors an averaged force arises. This force is defined by pulsed character of radiation and proportional to the intensity but not to gradient of intensity. It is shown that radiation losses are of little importance in the transverse plane but may considerably act on the longitudinal motion of electron.
\end{abstract}

Key words and phrases: relativistic electron, intense laser pulse, paraxial approximation, Gaussian beam, radiation friction

\section{Introduction}

When a relativistic charged particle moves in an electromagnetic field, radiation friction forces can play a significant role [1-3]. Various aspects of the radiation friction forces problem, including the refinement of the classical Lorentz-Abraham-Dirac expression, were discussed in [4-7]. The problem of radiation losses has become particularly relevant in connection with the creation of powerful laser radiation sources [8-10]. The charged particle motion pattern substantially depends on the properties of electromagnetic radiation. In the case of a sufficiently weak radiation intensity, the parameter $g=\frac{e|E|}{\omega m c}$, which is the ratio of the particle momentum oscillations amplitude in the wave field to a rest momentum, is small: $g \ll 1$. Here, $|E|$ is the amplitude of the electric wave field, $\omega$ is the wave frequency, $e$ is the charge, $m$ - the mass of the particle, $c$ is the light speed in vacuum. Availability of

(C) Dobrova E. V., Milantiev V.P., 2019 
a small parameter allows one to find a solution to the equations of motion for a particle in the form of expansions over this parameter and averaging over fast oscillations. When a particle moves in the high-power laser radiation field which is pulsed by its nature and of high intensity, the parameter $g$ becomes large. In the case of an electron, this parameter is represented as

$$
g=0.85 \cdot 10^{-9} \lambda \sqrt{I}
$$

where $I\left[W / \mathrm{cm}^{2}\right]$ is the laser pulse intensity, and $\lambda[\mu \mathrm{m}]$ is the wavelength. This shows that the parameter $g \geqslant 1$ at an intensity of $I \geqslant 10^{18} \mathrm{~W} / \mathrm{cm}^{2}$. Intensities of such an order and even higher by their value are well achieved in modern laser devices. In this case, a solution of the equations of motion for a particle in the form of expansions in the parameter (1) is impossible. Therefore, they usually use numerical methods for solving the problem. Analytical solution turns out to be possible if propagation of laser radiation is adequately described within the paraxial approximation in the form of Gaussian beams [11-17]. In this approximation, there is a small parameter

$$
\mu=\frac{2}{k a}=\frac{a}{z_{r}} \ll 1,
$$

where $a$ is the laser beam radius at focus (waist of the laser beam), $z_{r}=k a^{2} / 2$ is the Rayleigh length which determines the diffraction divergence of the laser beam, $k=2 \pi / \lambda$ is the wave number, and $\lambda$ is the radiation wavelength. The presence of such a small parameter (2) allows finding the solution of the equations of motion for a particle in the form of expansions in this parameter and averaging over fast oscillations of the radiation field. In the case of sufficiently long pulses, the radiation field vectors in the zero approximation of the expansion of Maxwell's equations in parameter (2) are represented in the form of Gaussian beams of different modes as a solution to the parabolic equation $[11,14]$. Upon that, the longitudinal components of the field vectors in the form of first-order corrections, and the corrections to the transverse components of the field vectors in the form of second-order quantities arise. In the case of sufficiently short pulses, the length of which is of the order of the beam waist, the corrections to the transverse components of the field vectors are first order quantities [13,17]. These amendments were not taken into account in papers [8-10]. Note that in the case of highly-focused laser radiation, paraxial approximation is inapplicable [18].

This work is devoted to the study of a relativistic electron motion in the field of high-power pulsed laser radiation in the form of a Gaussian beam with the circularly polarized basic mode, taking into account first-order corrections to field vectors and radiation friction force.

\section{Initial equations and problem statement}

In case of laser radiation with the circularly polarized basic mode, as a zero approximation of expansions in a small parameter, the field vectors are described by the formulas [17]: 


$$
\begin{aligned}
& E_{x}^{0}=\frac{f E_{x}(0)}{\sqrt{1+Z^{2}}} e^{\frac{-\rho^{2}}{1+Z^{2}}} \cos (\varphi+\theta)=A \cos (\varphi+\theta), \\
& E_{y}^{0}=-\frac{f E_{x}(0)}{\sqrt{1+Z^{2}}} e^{\frac{-\rho^{2}}{1+Z^{2}}} \sin (\varphi+\theta)=-A \sin (\varphi+\theta) .
\end{aligned}
$$

In the formulas written out: $\theta=\omega\left(\frac{z}{c}-t\right)$ is the "fast" wave phase, $\varphi(\rho, Z)=\frac{\rho^{2} Z}{1+Z^{2}}-\arctan (Z)$ is the slow phase, $f(\sigma)$ is the function which takes into account the pulsed nature of the radiation, and where the parameter $\sigma=\frac{\left(t-\frac{z}{c}\right)}{\Delta t}$, and $\Delta t$ is pulse duration. Dimensionless quantities are used for the longitudinal coordinate $Z=\frac{z}{z_{r}}$, the distance from the Gaussian beam axis in the transverse plane is $\rho=\frac{r}{a}$, where $r=\sqrt{x^{2}+y^{2}}$. In the first approximation for expansions of the field vectors with respect to the small parameter $\mu$, not only the longitudinal field components, but also corrections to the transverse components arise in the case of sufficiently short pulses [17]:

$$
\begin{aligned}
E_{x}^{1}= & -\frac{\lambda Z}{2 \pi c \Delta t} \frac{E_{x}(0) f^{\prime}}{\left(1+Z^{2}\right)^{3 / 2}} \sqrt{\left(1-\rho^{2}\right)^{2}+Z^{2}} e^{\frac{-\rho^{2}}{1+Z^{2}} \times} \\
& \times \cos \left(\arctan \frac{Z}{1-\rho^{2}}+\frac{\rho^{2} Z}{1+Z^{2}}-3 \arctan (Z)+\theta\right) \equiv-A_{1} \cos \left(\varphi_{1}+\theta\right), \\
E_{y}^{1}= & \frac{\lambda Z}{2 \pi c \Delta t} \frac{E_{x}(0) f^{\prime}}{\left(1+Z^{2}\right)^{3 / 2}} \sqrt{\left(1-\rho^{2}\right)^{2}+Z^{2}} e^{\frac{-\rho^{2}}{1+Z^{2}}} \times \\
& \times \sin \left(\arctan \frac{Z}{1-\rho^{2}}+\frac{\rho^{2} Z}{1+Z^{2}}-3 \arctan (Z)+\theta\right) \equiv A_{1} \sin \left(\varphi_{1}+\theta\right), \\
E_{z}^{1}= & \frac{2 f \rho E_{x}(0)}{k a\left(1+Z^{2}\right)} e^{\frac{-\rho^{2}}{1+Z^{2}}} \sin \left(\frac{Z \rho^{2}}{1+Z^{2}}-\arctan \frac{y}{x}-3 \arctan (Z)+\theta\right)
\end{aligned}
$$

Here $f^{\prime}(\sigma) \equiv \frac{\partial f}{\partial \sigma}$.

The relationship between the transverse components of the electric and magnetic vectors is determined by the relations:

$$
H_{x}=-E_{y}, \quad H_{y}=E_{x} .
$$

There is also a longitudinal component of the magnetic field of laser radiation:

$$
H_{z}^{1}=\frac{2 f \rho E_{x}(0)}{k a\left(1+Z^{2}\right)} e^{\frac{-\rho^{2}}{1+Z^{2}}} \sin \left(\frac{Z \rho^{2}}{1+Z^{2}}-\arctan \frac{y}{x}-3 \arctan (Z)+\theta\right) .
$$


The relativistic electron motion in an electromagnetic field is described by the equations:

$$
\frac{\mathrm{d} \vec{p}}{\mathrm{~d} t}=-e\left(\vec{E}+\frac{1}{m c \gamma}[\vec{p} \vec{H}]\right)+\vec{f}_{r}
$$

where $p$ is a particle momentum vector, $m$ is its mass; $\gamma=\sqrt{1+\frac{p^{2}}{m^{2} c^{2}}}$ is a relativistic factor (dimensionless particle energy), $\vec{f}_{r}$ is a radiation friction force.

The laser radiation field is given by formulas $(3-6)$. The constant magnetic field is directed along the $z$ axis: $\vec{H}_{0}=\left(0,0, H_{0}\right)$.

The system of equations (7) must be supplemented by an equation for the wave phase $\theta$ which is considered as a "fast" variable:

$$
\frac{\mathrm{d} \theta}{\mathrm{d} t}=-\omega\left(1-\frac{v_{z}}{c}\right) \equiv-\frac{\omega}{\gamma} G .
$$

Here $v_{z}$ is the longitudinal velocity of the particle; and the quantity $G$ has the following meaning

$$
G \equiv \gamma-\frac{p_{z}}{m c}
$$

It follows from equation (8) that the phase $\theta$ becomes a "slow" variable with $1-\frac{v_{z}}{c} \approx 0$. If $1-\frac{v_{z}}{c} \gg \mu$, then the phase $\theta$ can be considered as a "fast" variable over which it is possible to carry out averaging. This is assumed to perform in the future. According to the equations of motion (7), the value $G$ is described by the equation:

$$
\frac{\mathrm{d} G}{\mathrm{~d} t}=-\frac{e}{m c}\left(1-\frac{v_{z}}{c}\right) E_{Z} .
$$

This shows that in the first approximation, the quantity $G$ is on average preserved and is determined by the initial conditions. It is usually assumed that $G=1[1]$. From the equation (10) it follows that the quantity $G$ can be represented as an expansion:

$$
G=G_{0}+\mu G_{1}+\ldots
$$

Here $G_{0} \approx 1$ is the value averaged over the fast phase, and $G_{i}$ are quickly oscillating components of the quantity $G$.

It follows directly from the equations of motion for a particle that the relativistic factor satisfies the equation:

$$
\frac{\mathrm{d} \gamma}{\mathrm{d} t}=\frac{1}{\gamma(m c)^{2}} \vec{p}\left(-e \vec{E}+\vec{f}_{r}\right)
$$

The main task of this work is to obtain averaged equations for electron motion using the Bogolyubov's method [19], by averaging over the fast oscillations of laser radiation, taking into account the averaged radiation friction force. In their sense, the radiation friction forces are small compared 
with the effects of laser radiation. Therefore, we first consider the motion of a particle without taking radiation losses into account.

\section{Averaged equations of motion for a charged particle}

The initial equations of motion for a particle (7) contain members oscillating with the laser frequency, the amplitude of which is determined by parameter (1). In the conditions under consideration, this parameter is large. Therefore, it is impossible to carry out averaging according to the Bogolyubov's method [19] using expansions in the parameter $g$. However, the equations of motion also contain parameter (2) which is small. In principle, this allows to seek a solution in the form of expansions in the parameter $\mu$. However, it is necessary that the quickly oscillating members have small amplitude in order to apply the averaging method. This can be achieved if we firstly exclude in the equations of motion the quickly oscillating members with large amplitudes $[20,21]$. In this regard, we will make the replacement of the transverse components of the particle momentum vector, which is convenient to use in the complex form:

$$
p=\pi+C e^{i(\varphi+\theta)} .
$$

Here, $p=p_{x}+i p_{y}$ is the complex transverse momentum of a particle, and $\pi$ is the complex generalized momentum. Substituting (13) into system (7), we obtain the equation:

$$
\begin{aligned}
\frac{\mathrm{d} \pi}{\mathrm{d} t}+\frac{\mathrm{d} C}{\mathrm{~d} t} e^{i(\varphi+\theta)}+i C \frac{\mathrm{d} \theta}{\mathrm{d} t} e^{i(\varphi+\theta)}+i C \frac{\mathrm{d} \varphi}{\mathrm{d} t} e^{i(\varphi+\theta)}= \\
-e\left(1-\frac{v_{z}}{c}\right) A e^{i(\varphi+\theta)}+\frac{i \omega_{c o}}{\gamma}\left(\pi+C e^{i(\varphi+\theta)}\right)\left(1+\frac{H_{z}}{H_{0}}\right) .
\end{aligned}
$$

In the equation (14) the first member on the right is quickly oscillating with large amplitude. It can be eliminated by appropriate selection of the amplitude $C$ in the transformation (13). In its meaning, the amplitude $C$ must be determined by the amplitude of the radiation field in zero approximation. Then, equating the members of zero approximation in the equation (14) considering equation (8) and expansion (11), we get:

$$
C=\frac{-i e A}{\omega+\frac{\omega_{c o}}{G_{0}}}
$$

Here $\omega_{c o}=\frac{e H_{0}}{m c}$ is a classic cyclotron frequency. Thus, for the found value of $C$, equation (14) for the variable $\pi$ contains only members of the first order in the parameter $\mu$. We can obtain the following expression from the definition of the relativistic factor, taking into account (9)

$$
\gamma=\frac{1+G^{2}+\frac{p_{\perp}^{2}}{m^{2} c^{2}}}{2 G}
$$


where $p_{\perp}^{2}=p_{x}^{2}+p_{y}^{2}$. Given the replacement (13), we get

$$
p_{\perp}^{2}=\pi_{\perp}^{2}+\bar{C}^{2}-2 \bar{C} \pi_{\perp} \sin (\varphi+\theta-\beta) .
$$

Here: $\pi_{\perp}^{2}=\pi_{x}^{2}+\pi_{y}^{2}, \pi_{x}=\pi_{\perp} \cos \beta, \pi_{y}=\pi_{\perp} \sin \beta$.

The value of $\bar{C}=\frac{-e A}{\omega+\omega_{c o} / G_{0}}$ represents the amplitude of electron momentum oscillations in a field of laser radiation propagating along a constant magnetic field. Amplitude $A$ is determined by formulas (3). After a series of transformations, formula (16) takes the form:

$$
\gamma=\frac{1}{2 G_{0}}+\frac{G_{0}}{2}+\frac{\bar{C}^{2}}{2 G_{0} m^{2} c^{2}}+\frac{G_{1}}{2}-\frac{\bar{C} \pi_{\perp} \sin (\varphi+\theta-\beta)}{G_{0} m^{2} c^{2}}-\frac{\bar{C}^{2} G_{1}}{2 G_{0}^{2} m^{2} c^{2}} .
$$

Here the expansion $\frac{1}{G_{0}+G_{1}} \approx \frac{1}{G_{0}}\left(1-\frac{G_{1}}{G_{0}}\right)$ is taken into account. It is also assumed that $\pi_{\perp} \ll \bar{C}$. This is a natural limitation in particle acceleration problems. From the formula (18) it follows that the relativistic factor contains "constant" and fast oscillating parts:

$$
\gamma=\Gamma+\gamma_{1}
$$

where

$$
\Gamma=\frac{1+G_{0}^{2}+\frac{\bar{C}^{2}}{m^{2} c^{2}}}{2 G_{0}}, \quad \gamma_{1}=\frac{G_{1}}{2}-\frac{\bar{C}^{2} G_{1}}{2 G_{0}^{2} m^{2} c^{2}}-\frac{\bar{C} \pi_{\perp} \sin (\varphi+\theta-\beta)}{G_{0} m^{2} c^{2}} .
$$

Let us further consider the equation of longitudinal motion from system (7):

$$
\begin{aligned}
\frac{\mathrm{d} p_{z}}{\mathrm{~d} t}=e E_{z}^{1}+\frac{e}{m c \gamma}\left\{p_{x}\left[\frac{f E_{x}(0)}{\sqrt{1+Z^{2}}} e^{\frac{-\rho^{2}}{1+Z^{2}}} \cos (\varphi+\theta)+H_{y}^{1}\right]-\right. \\
\left.-\left[\frac{f E_{x}(0)}{\sqrt{1+Z^{2}}} e^{\frac{-\rho^{2}}{1+Z^{2}}} \sin (\varphi+\theta)+H_{x}^{1}\right] p_{y}\right\} .
\end{aligned}
$$

After a series of transformations we get:

$$
\begin{gathered}
\frac{\mathrm{d} p_{x}}{\mathrm{~d} t}=e \frac{2 f \rho E_{x}(0)}{k a\left(1+Z^{2}\right)} e^{\frac{-\rho^{2}}{1+Z^{2}}} \sin \left(\varphi-\arctan (Z)-\arctan \frac{y}{x}+\theta\right)+ \\
+\frac{2 e}{m^{2} c^{2}+G_{0}^{2} m^{2} c^{2}+\bar{C}^{2}}\left[G_{0}-\frac{G_{0}^{2} G_{1} m^{2} c^{2}-\bar{C}^{2} G_{1}-2 G_{0} \bar{C} \pi_{\perp} \sin (\varphi+\theta-\beta)}{m^{2} c^{2}+G_{0}^{2} m^{2} c^{2}+\bar{C}^{2}}\right] \times \\
\quad \times\left[A \pi_{\perp} \cos (\varphi+\theta+\beta)-A_{1} \pi_{\perp} \cos \left(\varphi_{1}+\theta+\beta\right)-\right. \\
-A \bar{C} \sin 2(\varphi+\theta)+A_{1} \bar{C} \sin \left(\arctan \frac{Z}{1-\rho^{2}}-2 \arctan (Z)\right)+
\end{gathered}
$$




$$
\left.+A_{1} \bar{C} \sin \left(\frac{2 \rho^{2} Z}{1+Z^{2}}-4 \arctan (Z)+\arctan \frac{Z}{1-\rho^{2}}+2 \theta\right)\right] .
$$

We omit the members containing $\pi_{\perp}$, and select the members that are independent on the fast phase $\theta$. As a result, we obtain the averaged equation of the longitudinal motion:

$$
\begin{aligned}
& \frac{\mathrm{d} \bar{p}_{z}}{\mathrm{~d} t}=\frac{e^{2} \lambda_{0} Z}{2 m \pi c^{2} \Delta t \Gamma} \frac{E_{x}^{2}(0) f f^{\prime}}{\left(1+Z^{2}\right)^{2}} \frac{1}{\omega+\omega_{c o} / G_{0}} \sqrt{\left(1-\rho^{2}\right)^{2}+Z^{2}} e^{\frac{-2 \rho^{2}}{1+Z^{2}}} \times \\
& \times \sin \left(\arctan \frac{Z}{1-\rho^{2}}-2 \arctan (Z)\right) .
\end{aligned}
$$

Here $\bar{p}_{z}$ is the average value for the longitudinal momentum of the particle. The right side of this equation, which has the first order of smallness in the parameter $\mu$, describes the averaged force with which high-power pulsed laser radiation acts on an electron in the direction of the magnetic field. A similar expression was obtained in [15] without taking into account the external magnetic field. However, due to the large difference between the cyclotron frequency and the laser radiation frequency, the influence of an external magnetic field is insignificant. As can be seen from (22), the averaged force is due to the pulsed nature of the radiation: $f^{\prime} \equiv \frac{\partial f}{\partial \sigma} \neq 0$.

\section{Radiation friction force}

The radiation friction force problem is one of the frequently discussed in the literature [3-10]. In $[6,7]$, some shortcomings were noted and refinements of the classical expression for the radiation friction force were made $[1,2]$. In the present work, the classical Lorenz-Abraham-Dirac formula is used for the radiation friction force [1-3]:

$$
\overrightarrow{f_{f r}}=\frac{2 e^{4}}{3 m^{2} c^{4}}\left\{[\vec{E} \vec{H}]+\frac{1}{c}[\vec{H}[\vec{H} \vec{v}]]\right\}-\frac{2 e^{4} \gamma}{3 m c^{3}} \vec{v}\left\{\left(\vec{E}+\frac{1}{c}[\vec{v} \vec{H}]\right)^{2}-\frac{1}{c^{2}}(\vec{E} \vec{v})^{2}\right\} .
$$

For the longitudinal motion, the radiation friction force when electron is moving in the laser field (3), taking into account the transformation (13), is represented as;

$$
\begin{aligned}
f_{z}= & \frac{2 e^{4}}{3 m^{2} c^{4}}\left\{A^{2} \cos ^{2}(\varphi+\theta)+A^{2} \sin ^{2}(\varphi+\theta)+\frac{H_{0}}{c m \gamma}\left[-A \bar{C} \sin ^{2}(\varphi+\theta)+\right.\right. \\
& \left.\left.+A \bar{C} \cos ^{2}(\varphi+\theta)\right]-\frac{p_{z}}{m c \gamma}\left[A^{2} \cos ^{2}(\varphi+\theta)+A^{2} \sin ^{2}(\varphi+\theta)\right]\right\}- \\
- & \frac{2 e^{4} \gamma}{3 m^{2} c^{3}} p_{z}\left\{A^{2} \cos ^{2}(\varphi+\theta)+A^{2} \sin ^{2}(\varphi+\theta)-\frac{2}{m c \gamma}\left[A \bar{C} H_{0} \sin ^{2}(\varphi+\theta)-\right.\right. \\
& \left.\quad-A \bar{C} H_{0} \cos ^{2}(\varphi+\theta)+p_{z}\left(A^{2} \cos ^{2}(\varphi+\theta)+A^{2} \sin ^{2}(\varphi+\theta)\right)\right]-
\end{aligned}
$$




$$
\begin{array}{r}
-\frac{1}{c^{2} m^{2} \gamma^{2}}\left[A^{2} \bar{C}^{2} \sin ^{4}(\varphi+\theta)+A^{2} \bar{C}^{2} \cos ^{4}(\varphi+\theta)-2 A \bar{C} H_{0} p_{z} \sin ^{2}(\varphi+\theta)+\right. \\
\left.\left.+2 A \bar{C} H_{0} p_{z} \cos ^{2}(\varphi+\theta)+2 A^{2} \bar{C}^{2} \sin ^{2}(\varphi+\theta) \cos ^{2}(\varphi+\theta)\right]\right\}
\end{array}
$$

Averaging over the fast oscillations of the radiation field and holding only the main members, we obtain the expression for the radiation friction force in the direction of the laser pulse propagation:

$$
\begin{gathered}
f_{a v z}=2 e^{4} \frac{f^{2} E_{x}^{2}(0)}{3 m^{2} c^{4}\left(1+Z^{2}\right)} e^{\frac{-2 \rho^{2}}{1+Z^{2}}}\left\{1-\frac{\bar{p}_{Z}}{m c \Gamma}\right\}-2 \frac{f^{2} E_{x}^{2}(0) e^{4} \Gamma}{3 m^{2} c^{3}\left(1+Z^{2}\right)} e^{\frac{-2 \rho^{2}}{1+Z^{2}}} \bar{p}_{Z} \times \\
\times\left\{1-\frac{2 \bar{p}_{Z}}{m c \Gamma}-\frac{e^{2} f^{2} E_{x}^{2}(0)}{m^{2} c^{2} \Gamma^{2}\left(1+Z^{2}\right)\left(\omega-\frac{\omega_{c o}}{G_{0}}\right)^{2}} e^{\frac{-2 \rho^{2}}{1+Z^{2}}}\right\} .
\end{gathered}
$$

Expression (24) is still quite complicated. For a rough estimate, we will assume that the particle velocity is close enough to the velocity of light. Then the main member of this expression is $-\frac{2 A^{2} e^{4} \Gamma}{3 m^{2} c^{3}} \bar{p}_{Z}$. From here, it can be concluded that the radiation reaction force can be sufficiently large in the field of high-intensity laser radiation. The calculations showed that the averaged transverse components of the radiation friction force are absent:

$$
f_{\text {avx }}=0, \quad f_{\text {avy }}=0 .
$$

This means that when a particle is moving in the transverse plane, the radiation losses on average play no role. When a particle is moving in the direction of radiation propagation, they can be significant in the field of super-intense laser radiation.

\section{Conclusion}

An expression for the averaged force acting on a relativistic electron in the field of an intense short laser pulse propagating along an external constant magnetic field is obtained. This force is due to the pulsed nature of the radiation, the description of which requires the inclusion of first-order members in expansions with respect to a small parameter of the paraxial approximation. It was taken into account that in the case of high-power laser radiation, the ratio of the amplitude of the particle oscillatory velocity in a wave field to the velocity of light can be large. To use the averaging over the fast oscillations of laser radiation, the transverse components of the particle momentum vector have been transformed. Thus, in the equations of motion, quickly oscillating members with large amplitude determined by the radiation field were eliminated. An expression was obtained for the averaged radiation friction force determined by the classical Lorentz-Abraham-Dirac formula. It was shown that this force in the transverse plane is zero and can be significant in the direction of laser pulse propagation. 


\section{Acknowledgments}

The publication has been prepared with the support of the "RUDN University Program 5-100".

\section{References}

1. L. D. Landau, E. M. Lifshitz, Field theory [Teoriya polya], Nauka, Moscow, 1988, in Russian.

2. J. D. Jackson, Classical electrodynamics, J. Wiley, NY-L., 1962.

3. V. L. Ginzburg, Theoretical physics and astrophysics [Teoreticheskaya fizika i astrofizika], Nauka, Moscow, 1975, in Russian.

4. N. P. Klepikov, Radiation damping forces and radiation from charged particles, Soviet Physics Uspekhi 28 (1985) 506-520. doi:10.1070/PU1985v028n06ABEH005205.

5. V. S. Krivitskii, V. N. Tsytovich, Average radiation-reaction force in quantum electrodynamics, Soviet Physics Uspekhi 234 (1991) 250-258. doi:10.1070/PU1991v034n03ABEH002352.

6. G. F. Efremov, Radiative damping of a relativistic electron in classical electrodynamics, Journal of Experimental and Theoretical Physics 89 (1998) 899-904. doi:10.1134/1.558738.

7. I. V. Sokolov, Renormalization of the Lorentz-Abraham-Dirac equation for radiation reaction force in classical electrodynamics, Journal of Experimental and Theoretical Physics 109 (2009) 207-212. doi:10.1134/S1063776109080044.

8. A. L. Galkin, Dynamics of an electron in a relativistically intense laser field including radiation reaction, Journal of Experimental and Theoretical Physics 115 (2012) 201-207. doi:10.1134/S1063776112070072.

9. A. V. Bashinov, A. A. Gonoskov, A. V. Kim, M. Marklund, G. Mourou, A. M. Sergeev, Electron acceleration and emission in a field of a plane and converging dipole wave of relativistic amplitudes with the radiation reaction force taken into account, Quantum Electronics 43 (2013) 291-299. doi:10.1070/QE2013v043n04ABEH015101.

10. K. Seto, H. Nagamoto, J. Koga, K. Mima, Equations of motion with radiation reaction in ultrarelativistic laser-electron interactions, Physics of Plasmas 18 (2011) 123404. doi:10.1063/1.3663843.

11. L. W. Davis, Theory of electromagnetic beams, Physical Review A 19 (1979) 1177-1179. doi:10.1103/PhysRevA.19.1177.

12. D. Bauer, P. Mulser, W. Steeb, Relativistic ponderomotive force, uphill acceleration and transition to chaos, Physical Review Letters 75 (1995) 4622-4625. doi:10.1103/PhysRevLett.75.4622.

13. B. Quesnel, P. Mora, Theory and simulation of the interaction of ultraintense laser pulses with electrons in vacuum, Physical Review E 58 (1998) 3719-3732. doi:10.1103/PhysRevE.58.3719.

14. A. M. Goncharenko, Gaussian light beams [Gaussovy puchki sveta], Nauka i tekhnika, Minsk, 1997, in Russian.

15. D. R. Bituk, M. V. Fedorov, Relativistic ponderomotive forces, Journal of Experimental and Theoretical Physics 89 (1999) 640-646. 
16. N. B. Narozhny, M. S. Fofanov, Scattering of relativistic electrons by a focused laser pulse, Journal of Experimental and Theoretical Physics 90 (2000) 753-768. doi:10.1134/1.559160.

17. V. P. Milant'ev, S. P. Karnilovich, Y. N. Shaar, Description of high-power laser radiation in the paraxial approximation, Quantum Electronics 45 (2015) 1063-1068. doi:10.1070/QE2015v045n11ABEH015800.

18. S. G. Bochkarev, V. Y. Bychenkov, Acceleration of electrons by tightly focused femtosecond laser pulses, Quantum Electronics 37 (2007) 273-284. doi:10.1070/QE2007v037n03ABEH013462.

19. N. N. Bogoljubov, Y. A. Mitropolskij, Asymptotic methods in the theory of nonlinear oscillations [Asimptoticheskiye metody v teorii nelineynykh kolebaniy], Nauka, Moscow, 1974, in Russian.

20. V. P. Milant'ev, A. J. Castillo, On the theory of the relativistic motion of a charged particle in the field of intense electromagnetic radiation, Journal of Experimental and Theoretical Physics 116 (2013) 558-566. doi:10.1134/S1063776113040067.

21. A. J. Castillo, V. P. Milant'ev, Relativistic ponderomotive forces in the field of intense laser radiation, Technical Physics 59 (2014) 1261-1266. doi:10.1134/S1063784214090138.

\section{For citation:}

E. V. Dobrova, V. P. Milantiev, On the radiation losses during motion of an electron in the field of intense laser radiation, Discrete and Continuous Models and Applied Computational Science 27 (2) (2019) 133-142. DOI: 10.22363/26584670-2019-27-2-133-142.

\section{Information about the authors:}

Ekaterina V. Dobrova (Russian Federation) - student of Institute of Physical Research and Technology of Peoples' Friendship University of Russia (RUDN University) (e-mail: dobrova03@icloud.com, phone: $+7(499) 2483057)$

Vladimir P. Milantiev (Russian Federation) - Professor, Doctor of Physical and Mathematical Sciences, professor of Institute of Physical Research and Technology of Peoples' Friendship University of Russia (RUDN University) (e-mail: vmilant@mail.ru, phone: +7(499) 2483057, Scopus Author ID: 6506148930) 